\title{
The Video Collaboratory as a Learning Environment
}

\author{
Vikash Singh, Sarah Abdellahi, Mary Lou Maher and Celine Latulipe \\ Department of Software and Information System \\ University of North Carolina at Charlotte \\ Charlotte, NC, USA
}

\{vsingh7, sabdella, m.maher, clatulip\}@uncc.edu

\begin{abstract}
Video usage in educational environments has increased significantly with the expansion of online learning opportunities. However, current video-based applications used for learning are designed for passive consumption of video content and do not support small-group collaboration. Specifically, they lack interaction affordances for active and collaborative learning through video materials. In this paper, we present the affordances and user experience of a web-based video application called Video Collaboratory that is designed with multiple affordances for group-based learning around video content. We surveyed students and analyzed the use of the Video Collaboratory in an HCI course. Students found the Video Collaboratory helpful because they could learn from their peers. Students also used the application beyond the activities required for credit.
\end{abstract}

\section{Keywords}

Computer-supported collaborative learning, flipped courses, video annotation, collaborative computing

\section{INTRODUCTION}

The growth of Massive Open Online Courses (MOOCs) in recent years shows the huge potential of video as a medium for education. Video lectures and tutorials are the primary method of instruction in MOOCs, delivered either through YouTube as in the case of Udacity, or native video applications as in Coursera and edX. Besides MOOCs, other approaches for online learning include blended or hybrid methods that involve face-to-face interactions among students in addition to online video-based instruction. The flipped classroom approach requires students to come to class after viewing the required materials so that they are ready to engage in learning activities [1,10,12]. Learning materials such as lectures and presentations are delivered to students through an alternate medium such as podcasts or videoon-demand services $[5,10,15,17]$. Students can view these materials on their own time prior to coming to the class, allowing the use of class time for active learning. In-class activities include quizzes, collaborative learning exercises, and working in a group towards a project goal. Various strategies for flipped classroom learning in CS education are described in Maher et al [13].

Permission to make digital or hard copies of part or all of this work for personal or classroom use is granted without fee provided that copies are not made or distributed for profit or commercial advantage and that copies bear this notice and the full citation on the first page. Copyrights for third-party components of this work must be honored. For all other uses, contact the Owner/Author.

Copyright is held by the owner/author(s).

SIGCSE '16, March 02-05, 2016, Memphis, TN, USA

ACM 978-1-4503-3685-7/16/03.

DOI: http://dx.doi.org/10.1145/2839509.2844588
A challenge in using video in online education environments is the inability to tailor video environments for collaborative learning. In the case of MOOCs, the video lectures are not directly connected to the discussion about the video. For example, the students on MOOCs generally post their comments about video lectures in a separate forum or send an email to their instructors with their question. Such disjointed interaction may create confusion, waste time and increase miscommunication. In some MOOC platforms such as Udacity, the users are presented with formative quizzes to test users' learning but these assessments are targeted to individuals and lack a collaborative approach to learning. The lack of collaborative engagement leads to the individual user passively consuming the videos [11] rather than actively interacting with the video as a group.

In this paper, we discuss some of the video-based educational platforms and demonstrate a lack of affordances for collaborative learning through video. Then we present the Video Collaboratory application, a tool for video-centered collaborative learning. We used the Video Collaboratory for collaborative learning around video lectures in our HCI course in the Spring 2015 semester and we present our analysis of student perceptions and usage of the tool in this course.

\section{RELATED WORK}

Collaboration and peer learning are important components of the learning process. Through collaboration and peer learning, students develop critical thinking skills and discover important issues found by other students [18]. Collaborative learning allows students to interact with their peers to achieve a common learning goal [14]. A three years study of students' performance on final exam scores between control and experimental student groups showed higher scores for students who completed collaborative exercises than those who were in a traditional lecture-based course that focused on individual learning [4].

A review of active learning methods clearly suggests that, like other instructional practices, collaborative learning has strong advantages [14]. Many groups have explored the idea of using video annotation interfaces as a support tool for educators to enhance active learning. However, most of the education-specific video software systems lack support for collaboration among students and between students and instructor.

In the education domain, there are many popular web-based or standalone video interfaces used for content delivery. Some popular examples are Coursera ${ }^{1}$, edX $^{2}$, Udacity $^{3}$, GoReact $^{4}$, and

\footnotetext{
${ }^{1}$ http://coursera.com

2 http://edx.org

3 http://udacity.com

${ }^{4}$ http://goreact.com
} 
edPuzzle ${ }^{5}$. Other examples include MRAS [3], videoANT [8], and TrACE [7]. Most of these applications focus on content delivery and have their own novel design features to support communication between instructors and students. These features not only enhance the video watching experience by supporting different ways of video navigation (edX, videoANT) but also increase interactivity with the lecture videos by allowing instructors to embed quizzes in the lectures (Udacity, edPuzzle). Other features such as the ability to evaluate a video with rating scales (GoReact) and add text annotations to different parts of the video (videoANT, GoReact, TrACE) contribute to interactions between the instructors and the students.

However, none of these tools are designed for collaborative sensemaking, an important component of the learning process. Many applications support embedding quizzes inside a video lecture but the process of embedding quizzes is done before student consumption of video content and although it adds some interactivity to the video, it doesn't support student-student or student-instructor collaboration (Udacity, edPuzzle). Similarly, video annotations are supported for videos more for self-reference (edX) and are not synchronously or asynchronously available to other users for collaboration. Some systems support sharing of annotations through email (videoANT, MRAS) but using a separate channel for sharing annotations makes the discussion disjointed from the video.

The lack of affordances for collaboration in these systems inhibits collaborative discussion and does not offer an engaging video experience. To address this need for a collaborative platform for video-centered discussion, we have designed and developed the Video Collaboratory system.

In the classic CSCW time-space matrix for groupware [9], collaboration among group members can happen synchronously or asynchronously depending on whether the group members are present at the same time or not. Similarly, whether the participants are co-located or collaborating remotely also affects the choice of groupware. Sense-making of an online lecture video is an asynchronous process where different students watch a video at their own time. Using the Video Collaboratory for sense-making of video lectures falls into the asynchronous-remote quadrant. In the next section, we discuss the various affordances in the Video Collaboratory system that allow collaboration among students.

\section{VIDEO COLLABORATORY}

The Video Collaboratory (VC) is a web-based platform that provides unique interaction techniques and a user experience that is customized towards enabling group collaboration around video (Figure 1). VC allows small groups to create text and sketch annotations on existing videos: enabling effective and creative collaboration centered around video artifacts. The Video Collaboratory has been developed through an iterative process following a research through design approach [19] where the design of the collaborative environment led to insights on affordances for collaboration around video content. A major contribution is an environment for collaboration that connects annotations and comments directly to video content and eliminates the need for a separate channel for discussion around a video.

\footnotetext{
${ }^{5} \mathrm{http}: / /$ edpuzzle.com
}

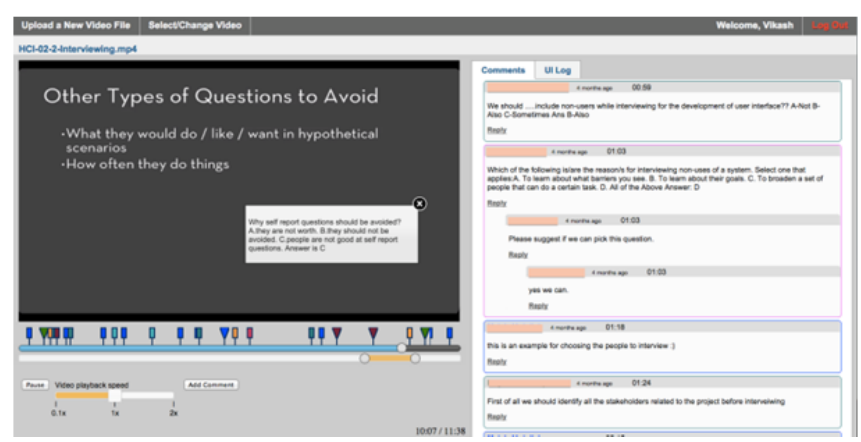

Figure 1. Video Collaboratory main screen.

\subsection{Affordances for Video-centered Collaboration}

The affordances for video-centered collaboration include:

\subsubsection{Context awareness}

Awareness and notifications are two important aspects of both synchronous and asynchronous collaboration [2]. While working with collaborators, it becomes important to know the context of the communication content to facilitate discussion [6]. In the case of video-centered collaboration, a video's timeline represented by a scrubber is an important signifier as it represents the full length of a video. In the Video Collaboratory, the markers on the scrubber provide affordances for recognizing the context of a comment or annotation, and are signifiers for the specific location on the video timeline for specific comments. When moving a mouse or other pointing device over a marker, the user has a preview of the comment at that point in time at a glance or can navigate to that point on the video by clicking. This feature is important for collaboration because while engaging with a video lecture it becomes easier to note interesting or important sections by noticing the density and distribution of comment markers on the video timeline.

\subsubsection{Contextual navigation}

In the Video Collaboratory, the video could be navigated through comments in the right panel or the markers on the timeline. When a user clicks on a comment on the panel to the right of the video window, the video relocates on the timeline and automatically loops to display annotations in context. Two different kinds of markers are used to signify whether the marker represents a comment on a frame (rectangular) or on a segment of video (inverted triangle). This contextual navigation is important for collaboration because it helps collaborators quickly interpret the meaning of comments from other group members by seeing the video segment that is the context for each comment.

\subsubsection{Color-coding}

A different color codes each member's comments, allowing collaborators to visually identify different members' comments from a list of comments. This facilitates collaboration as it allows users to quickly follow the activities of another group member.

\subsubsection{Threaded discussion}

Threaded commenting is an important design affordance for collaborative interaction. It facilitates discussion among collaborators by allowing replies to a posted comment. In the Video Collaboratory, the 'Reply' button at the bottom of each comment signifies that a threaded discussion is supported in the system. The threaded conversation retains the color-coding of 
different collaborators, clearly representing the participants in a discussion.

\subsubsection{Multi-modal annotations}

Multiple modalities allow users to collaborate around videos in different ways. Sketching on the video frame allows users to specify spatial context that is not possible with text comments. The sketching modality is important to collaboration because it allows users to communicate spatial information and to sketch ideas onto the content in the video.

\subsection{User Interface}

After launching the VC application, the user enters login details for authentication and identification so the source of comments can be associated with a specific individual. Each registered user has access to a set of assigned videos and can select one for viewing and interaction. The collection of videos is presented in a tree like structure where each root node represents a collection of videos assigned to a group of users for a course or project. When a root node is clicked, all the videos inside that folder are displayed in a tile format (Figure 2). The user can hover their cursor over any of the videos to get a preview of the video. The user can then click on any of the tiled videos to open the main screen of the Video Collaboratory for that video. The main screen of the application has a two-column layout. The left column contains the video panel and the right column contains the comments panel with all the comments posted on that video (Figure 1). The video controls are located in the first column below the video. The annotation tools and palette are on top of the video panel.

\subsection{User Interaction}

The Video Collaboratory supports a wide array of interactions with video. In addition to supporting the default controls for video such as a play/pause, toggle, and video scrubbing, it supports some novel interaction techniques that are specially designed for video-based collaboration. Some of the user interactions supported by the application are described next.

\subsubsection{Inserting Comments}

On the main screen, comments can be inserted on the video timeline in two different modalities by clicking the 'Add Comment' button. Once a user chooses to insert a comment, he can choose between text or sketch or a combination of both modalities. The comments can be added to a point (frame) or to a selected segment of any length of the video. Once a comment is submitted, it is added to the list of comments in the comments panel on the right and a color-coded marker appears over the video timeline showing the position of the inserted comment on the video timeline. A user can reply to a comment by clicking on the reply button at the bottom of each comment on the right panel.

\subsubsection{Exploring and Editing Comments}

Posted comments can be explored in two different ways. A user can click on any comment in the comments panel or on a colored marker on the video timeline. Either action moves the video playhead to the appropriate part of the video and loops the playback between 2 seconds prior and 2 seconds after the comment point. For segment comments, the video loops over the specified segment of the video. Comments related to that marker are then overlaid on the main video. Users can view, edit or delete their own comments, but can only view the comments of others.

\subsubsection{Annotations Preview}

When many collaborators use a video, the timeline could potentially become crowded with comments. If a user is looking to revisit a comment, or wants to get a quick sense of the types of comments found in one part of the video, she can hover her cursor over the comment markers. This helps the user browse and navigate without having to click on each individual annotation marker. Additionally, as a user hovers over a segment marker, the length of the segment is highlighted on the Segment Selector as a preview.

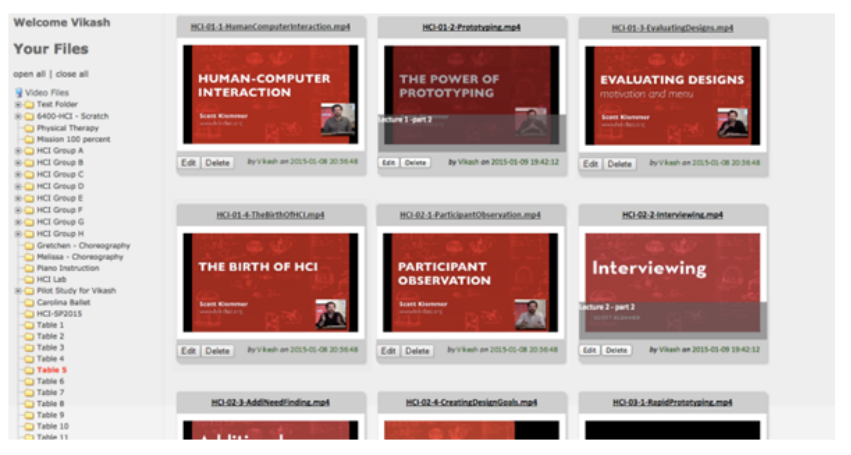

Figure 2. Tiled videos on video selection screen.
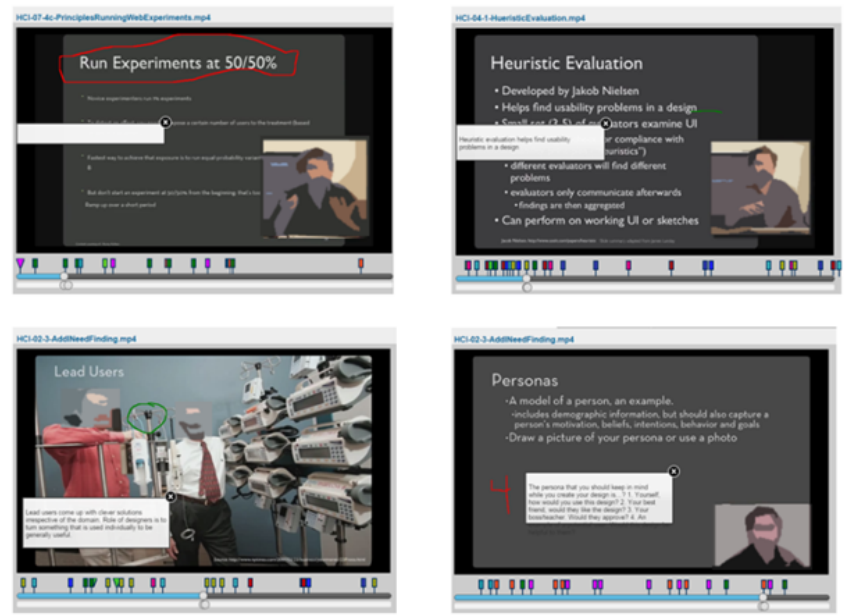

Figure 3. Examples of sketching on lecture videos by students.

\section{VIDEO COLLABORATORY USE IN A FLIPPED CLASS}

In the Spring 2015 semester, we used the Video Collaboratory system for the Human Computer Interaction course at our institution. The course was taught as a flipped class, with video lectures watched outside of class, and hands-on group activities during scheduled class time. There were 121 students enrolled in the class: 62 graduate students and 59 undergraduate students.

The students were placed in groups where each group was all undergraduate or graduate students. The students were divided into 14 groups with a minimum of 6 and a maximum of 9 students per group. We kept the undergraduates and graduate students separate for pedagogical reasons that were independent of the evaluation of the use of the VC. As we reported the usage of the VC separately for graduates and undergraduates, we noticed different levels of engagement with the lecture material, possibly due to different levels of maturity and attention to the course. In future use of the $\mathrm{VC}$, we plan to develop different strategies in creating assignments and activities around lecture videos for different levels of students. 


\subsection{Active Learning around Video Lectures}

The students in each group shared access to the same weekly lecture and therefore shared their comments and annotations through the Video Collaboratory. That is, each group had their own folder on the Video Collaboratory with their own private copy of the lecture videos. This design was purposeful: first, small group collaboration can help students feel more comfortable and less intimidated than they might if they had to post comments seen by 120 other students. Second, by limiting interaction around each video instance to a smaller group of people, the timeline would not become too cluttered with annotation markers. This makes it possible for students to see each other's markers and get to know each other's marker colors, helping to create a visual image of who is commenting and where. This would not be feasible with 120 different colors and hundreds of markers on a single video.

Each student was required to enter 3 comments on the assigned lecture each week to identify 3 multiple-choice questions as the significant content of the video lecture. Each group was then asked to reach a consensus on two questions to be sent to course TAs prior to the start of the class each week. These two questions from each of the 14 groups formed the question pool for that week's clicker quiz class activity. The TAs then selected 4-6 questions from the question pool and other resources to create that week's clicker quiz.

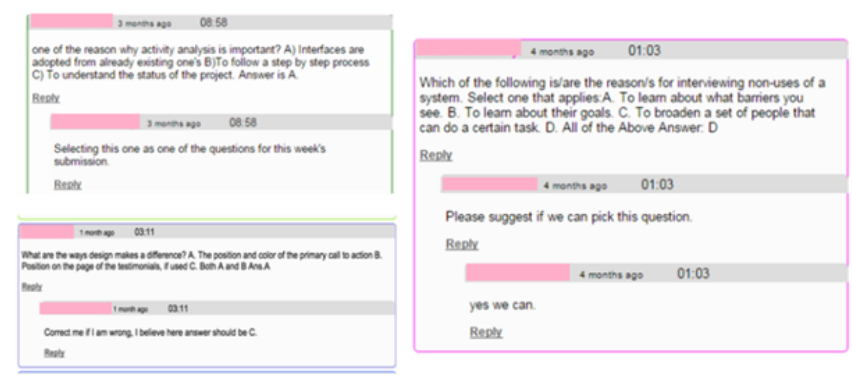

Figure 4. Examples of discussions among students about weekly quiz questions.

Students were motivated to produce the multiple-choice questions in 3 ways. First, groups with the best choice of questions during the semester were given extra credit. Second, each individual student had to participate in a clicker quiz with questions partly chosen among the aggregated questions submitted by the groups each week. These quizzes were graded as a part of the students' final grade and groups with the best choice of questions had the chance to answer questions designed by their own group in the clicker quiz. Third, the annotations, multiple choice questions, and comments from the entire class were collected and made available to the students as a study guide for the mid-term and final exams.

\subsection{Data Collection and Analysis}

We gathered two sets of data during the semester: survey data and VC usage data. The survey included questions about the students' perception of the flipped classroom learning experience. Some of the questions students answered in the survey are relevant to the $\mathrm{VC}$ and are shown in Table 1.

We conducted the same survey before the mid-term and then again before the final exam to see if student perceptions of the flipped classroom had changed. 110 students participated in the mid-term survey and 98 students completed the survey before the final exam.
Table 1. Survey results - Mean and median of responses on a 7 point Likert scale

\begin{tabular}{|c|l|l|l|}
\hline & $\begin{array}{l}\text { How } \\
\text { helpful } \\
\text { were the } \\
\text { online } \\
\text { videos } \\
\text { for } \\
\text { learning } \\
\text { HCI } \\
\text { methods? }\end{array}$ & $\begin{array}{l}\text { Other } \\
\text { students } \\
\text { helped me to } \\
\text { understand } \\
\text { the right } \\
\text { answers while } \\
\text { we were } \\
\text { discussing } \\
\text { quiz } \\
\text { questions. }\end{array}$ & $\begin{array}{l}\text { I helped } \\
\text { other } \\
\text { students to } \\
\text { understand } \\
\text { the right } \\
\text { answers } \\
\text { while we } \\
\text { were } \\
\text { discussing } \\
\text { quiz } \\
\text { questions. }\end{array}$ \\
\hline $\begin{array}{c}\text { Mid-term } \\
\text { Average } \\
\text { (Median) }\end{array}$ & 4.35 & 4.73 & 5.01 \\
\hline Final & $(4)$ & $(5)$ & $(5)$ \\
\hline $\begin{array}{l}\text { Average } \\
\text { (Median) }\end{array}$ & 4.03 & 4.44 & $(4)$ \\
\hline
\end{tabular}

The survey tool was developed to examine student perceptions of flipped classrooms and had a total of 24 questions. Here we only report on the 3 questions relevant to the use of the Video Collaboratory. The results for questions about the online lecture videos and the collaborative approach to quizzes are shown in Table 1. While there was a drop in the average value on the Likert scale for these questions, this difference is not significant. Students generally liked using the videos on the Video Collaboratory. They also liked the collaborative nature of the application that allowed them to prepare for the quizzes together. In the Video Collaboratory the students posted questions for each other and let other members of the group answer the questions. Many students used the Video Collaboratory application as a tool for creating references on the video for self-study. Some students used sketching to write answers of self-created quiz questions on the video as shown in Figure 3.

We gathered data on the use of comments, annotations, and replies in the VC. We aggregated the data into student groups. We looked specifically for evidence of collaboration on the VC for coming to consensus on choosing group questions. The reason for gathering this data is to compare the actual number of comments to the required number of comments as an indicator of the usability of the VC and as an indicator of the students' perceived value of video engagement. Since the VC supports more than just watching video lectures, the VC usage data also allows an analysis of how engaged the students are with the video lectures beyond a record of how much time they spent watching the video.

We found that the students added more comments on the videos than the amount required for the assignment. Since each group had up to 9 members, and each person was required to provide 3 comments per week, the maximum required number of comments per week is 27. As shown in Figure 5 and 6, most of the time groups added more comments than required for grades. In several cases, up to four times the expected number of comments was observed. The recorded data shows as many as 111 comments from a single group in one week. The analysis of the data on the number of comments on video lectures shows interesting patterns in students' use of the $\mathrm{VC}$, and is an indicator of student engagement in the lecture material. A visual representation of the 


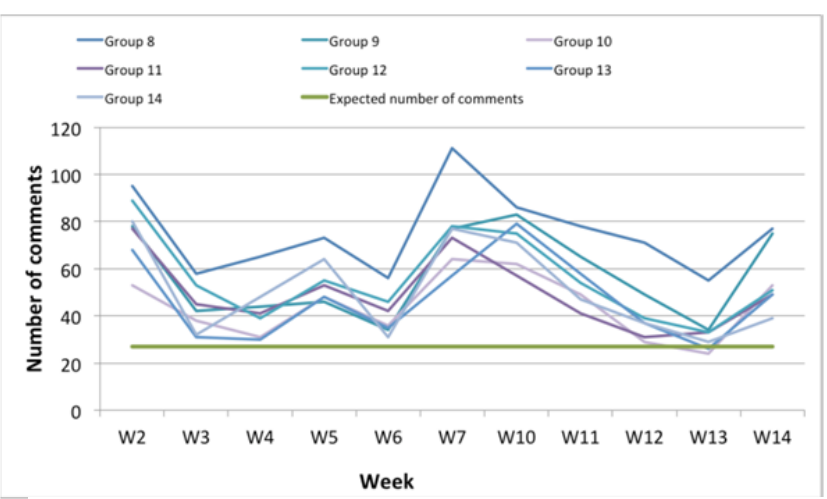

Figure 5. Number of comments posted by graduate groups on $\mathrm{VC}$ each week compared to the expected number in each assignment.

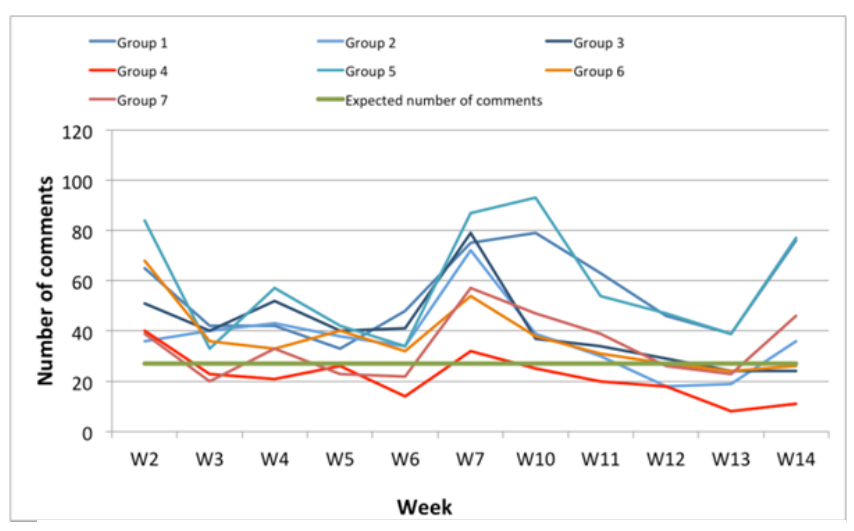

Figure 6. Number of comments posted by undergraduate groups on VC each week compared to the expected number by the assignment number in each assignment.

data on students' use of VC is available in Figure 5 for graduate students and Figure 6 for undergraduate.

The flat green line in Figures 5 and 6 represents the required number of comments for each week. While some groups did significantly more than required (groups $8,9,10,12,13,14$ ) and others did less (groups 4 and 7), all groups had a strong start at the beginning of the semester and a decrease in the amount of work after the second week. All groups had a significant increase in the amount of comments placed on the videos the week before and after the midterm exam, and the week before the final exam. The groups had fewer comments in the VC during weeks with project submission deadlines (weeks 6 and 13).

The significance of visualizing this usage data is the additional information it provides to the instructor about student preparation, engagement, and collaboration. Where most video platforms for online education provide the instructor with information about how many students viewed the video and for how long, the VC provides information about how much the students engaged with the video content by showing their comments and annotations.

We observed a significant difference in the amount of work done by graduate students in the class (groups 8 to 14) compared with the undergraduates (groups 1 to 7 ). While the graduate groups placed up to 4 times the expected number of comments on the videos, undergraduate groups moved closer to the required amount of work, and 2 groups (groups 4 and 7) didn't complete the required work in some weeks. Again, the significance of visualizing the difference between the graduate student groups and the undergraduate student groups is the feedback this provides to the instructor. The graduate students were more engaged with the lecture material than the undergraduates. This is important information when interacting with the students during in-class activities and when evaluating their projects. This analysis of the use of the VC could lead the instructor to be more strategic about assignments that would encourage a more uniform engagement with video content.

The extra comments posted by the groups on the videos were not limited to the quiz design assignments. The content of comments placed by the students also included:

- Finalizing the quiz questions and decision-making about the group submission.

- Highlighting, and summarizing important points mentioned in the videos.

- Discussing confusion about course material in the video and helping each other to understand the material.

While students had been required to post quiz questions on the $\mathrm{VC}$, using it as a medium for the three other purposes mentioned above was their own choice. Choosing the VC for communication about course material confusion and to ask peers for help supports the importance of context awareness in collaboration. Also, using the VC to highlight the important points supports both the importance of context awareness and contextual navigation as it allows them to review the course material by navigation through the comments and review the related video, in addition to providing the possibility of reviewing the points considered important by their peers.

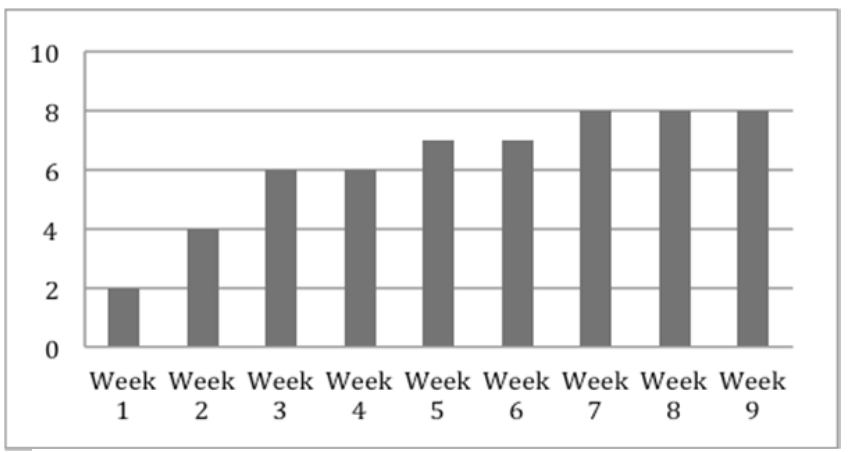

Figure 7. Number of groups using VC reply option for discussion around the assignment each week number by the assignment number in each assignment.

An indication of collaborative communication is the number of times the groups used the reply option. Figure 7 shows how many groups used the reply option each week. More groups used the VC as a collaborative communication media at the end of the semester compared with the start of the semester. While in the first week two groups were using the reply feature on the VC for discussion around the activity, after week 7, 8 groups had used that feature for discussion around the assignment. These groups did more than just enter their quiz questions on the videos, and used the $\mathrm{VC}$ communication features (commenting and replying) for choosing the group's best questions for submission each week. In the beginning of the semester the groups were using other communication channels such as email for exchanging thoughts about the group weekly assignment, but by the end of the semester more groups were relying on the commenting and reply features of the $\mathrm{VC}$ for conversation around the quiz questions, consensus 
and decision making, and clarifying misunderstandings. Figure 4 shows a few examples of these uses by some of the groups. This type of analysis of the annotations on educational video content could lead to more strategic use of video and activities around video.

\section{SUMMARY AND CONCLUSION}

Collaborative learning is a complex social process and any software environment intended to support collaborative learning should afford and encourage ideas from multiple viewpoints that can be further developed as students approach consensus [16]. The Video Collaboratory provides affordances to support this social process around video material. Students can watch a video, mark up and annotate different points and segments of the assigned videos. The Video Collaboratory not only allows students to reach a consensus on weekly tasks but also helps them create collective self-study material. The design of the $\mathrm{VC}$ has focused on asynchronous collaboration, and future developments include a signifier for presence of others viewing and annotating the same video as an affordance for synchronous collaboration.

Our analysis of the use of the VC in a flipped classroom learning environment shows that students engaged with the video content more than was required, implying that the students found value beyond that needed to get the credit for commenting on video content. The students' video comments and annotations also provided the instructor with a better understanding of student engagement with video lecture content since this data shows more than just the amount of time they watch the video: including data about their active note taking and communication with others around the video content.

The design of the features and affordances in the $\mathrm{VC}$ has focused on the collaborative user experience, assuming that the user is collaborating around video content. In the context of education, the $\mathrm{VC}$ user experience has focused on the student as user and instructional material as content. As we gain more experience with the use of $\mathrm{VC}$ in a learning environment, we are planning the development of the user experience for the instructor. The analysis of student engagement can lead to different educational strategies around online lectures, beyond embedding quizzes as is typical in current online learning environments. Our analysis of the student engagement with video content required extracting the logged data and performing an analysis outside the VC. Future versions of $\mathrm{VC}$ should provide this analysis as a built-in aid to the instructor.

\section{REFERENCES}

[1] Ellen R. Auster and Krista K. Wylie. 2006. Creating Active Learning in the Classroom: A Systematic Approach. Journal of Management Education 30, 2: 333-353.

[2] Ronald Baecker, Dimitrios Nastos, Ilona Posner, and Kelly Mawby. 1993. The user-centered iterative design of collaborative writing software. ACM Press, 399-405.

[3] D. Bargeron, A. Gupta, J. Grudin, and E. Sanocki. 1999. Annotations for streaming video on the web. CHI ' 99 Extended Abstracts, 278-279.

[4] Leland Beck and Alexander Chizhik. 2013. Cooperative learning instructional methods for CS1: Design, implementation, and evaluation. ACM Transactions on Computing Education (TOCE) 13, 3.

[5] Jacob Lowell Bishop and Matthew Verleger. 2013. The Flipped Classroom: A Survey of the Research. 120th ASEE Annual Conference.
[6] J. J. Cadiz, Anop Gupta, and Jonathan Grudin. 2000. Using Web Annotations for Asynchronous Collaboration Around Documents. Proceedings of the 2000 ACM Conference on Computer Supported Cooperative Work, ACM, 309-318.

[7] Brian Dorn, Larissa B. Schroeder, and Adam Stankiewicz. 2015. Piloting TrACE: Exploring Spatiotemporal Anchored Collaboration in Asynchronous Learning. Proceedings of the 18th ACM Conference on Computer Supported Cooperative Work, ACM, 393-403.

[8] Brad Hosack, Charles Miller, and David Ernst. 2009. VideoANT: Extending Video beyond Content Delivery through Annotation. Proceedings of World Conference on ELearning in Corporate, Government, Healthcare, and Higher Education 2009, AACE, 1654-1658.

[9] Robert Johansen. 1988. GroupWare: Computer Support for Business Teams. The Free Press, New York, NY, USA.

[10] Maureen J. Lage, Glenn J. Platt, and Michael Treglia. 2000. Inverting the Classroom: A Gateway to Creating an Inclusive Learning Environment. The Journal of Economic Education 31, 1: 30-43.

[11] Fei Xue Larissa Schroeder. 2013. Flipping three different mathematics courses: Common conclusions and plans for the future. MathAMATYC Educator 4, 2: 63-68.

[12] Kate Lockwood and Rachel Esselstein. 2013. The inverted classroom and the CS curriculum. Proceeding of the 44th ACM technical symposium on Computer science education, ACM, 113-118.

[13] Mary Lou Maher, Celine Latulipe, Heather Lipford, and Audrey Rorrer. 2015. Flipped Classroom Strategies for CS Education. Proceedings of the 46th ACM Technical Symposium on Computer Science Education, ACM, 218 223.

[14] Michael Prince. 2004. Does Active Learning Work? A Review of the Research. Journal of Engineering Education 93, 3: 223-231.

[15] Nancy M. Schullery, Robert F. Reck, and S. Schullery. 2011. Toward solving the high enrollment, low engagement dilemma: A case study in introductory business. International Journal of Business, Humanities and Technology 1, 2: 1-9.

[16] Gerry Stahl. 2000. A model of Collaborative KnowledgeBuilding. Fourth International Conference of the Learning Sciences, 70-77.

[17] Roxanne Toto and Hien Nguyen. 2009. Flipping the work design in an industrial engineering course. Proceedings of the 39th IEEE international conference on Frontiers in education conference, IEEE Press, 1066-1069.

[18] William M. Waite, Michele H. Jackson, Amer Diwan, and Paul M. Leonardi. 2004. Student Culture vs Group Work in Computer Science. Proceedings of the 35th SIGCSE Technical Symposium on Computer Science Education, ACM, 12-16.

[19] John Zimmerman, Jodi Forlizzi, and Shelley Evenson. 2007. Research Through Design As a Method for Interaction Design Research in HCI. Proceedings of the SIGCHI Conference on Human Factors in Computing Systems, ACM, 493-502. 